\title{
Reconnection in a slow Coronal Mass Ejection
}

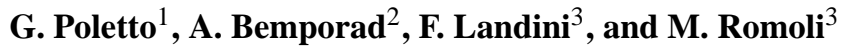 \\ ${ }^{1}$ INAF - Arcetri Astrophysical Observatory, Firenze, Italy \\ ${ }^{2}$ INAF - Astronomical Observatory, Pino Torinese, Torino, Italy \\ ${ }^{3}$ Dept. of Astronomy and Space Science, University of Firenze, Italy
}

Received: 8 October 2007 - Revised: 26 May 2008 - Accepted: 26 August 2008 - Published: 15 October 2008

\begin{abstract}
This paper aims at studying reconnection occurring in the aftermath of the 28 May 2004, CME, first imaged by the LASCO (Large Angle and Spectrometric Coronagraph) $\mathrm{C} 2$ at 11:06 UT. The CME was observed in White Light and UV radiation: images acquired by the LASCO C2 and $\mathrm{C} 3$ coronagraphs and spectra acquired by UVCS (Ultraviolet Coronagraph Spectrometer) allowed us to identify the level at which field lines, stretched outwards by the CME ejection, reconnect below the CME bubble. As the CME propagates outwards, reconnection occurs at increasingly higher levels. The process goes on at a low pace for several hours: here we give the profile of the reconnection rate vs. heliocentric distance over a time interval of $\approx 14 \mathrm{~h}$ after the CME onset, extending estimates of the reconnection rate to larger distances than previously inferred by other authors. The reconnection rate appears to decrease with time/altitude. We also calculate upper and lower limits to the density in the diffusion region between 4 and $7 R_{\odot}$ and conclude by comparing estimates of the classical and anomalous resistivity in the diffusion region with the value inferred from the data. The latter turns out to be $\geq 5$ order of magnitudes larger than predicted by classical or anomalous theories, pointing to the need of identifying the process responsible for the observed value.
\end{abstract}

Keywords. Solar physics, astrophysics, and astronomy (Flares and mass ejections) - Space plasma physics (Magnetic reconnection)

\section{Introduction}

Coronal mass ejections occur as a consequence of the disruption of a closed magnetic structure (Wagner, 1984). How this occurs has been modeled in different ways by many authors who invoked reconnection processes, independently of

Correspondence to: G. Poletto

(poletto@arcetri.astro.it) the precise features of the diverse scenarios (see, e.g. Antiochos et al., 1999; Lin and Forbes, 2000). In particular, catastrophe models predict the formation of a current sheet (CS) connecting the top of post-eruption loops to the lower part of the expanding CME bubble. Current sheets also appear in numerical simulations of CMEs (see, e.g., Riley et al., 2007; Bárta et al., 2007).

Nowadays the most popular CME model is the catastrophe model, which has been supported, over the last few years, by the identification of current sheets in the predicted position, i.e. between the loop arcade reforming below the CME and the CME core. The detection of CSs was perhaps contrary to expectations, as CSs were supposed to be so thin to be hardly observable at all. These observations are relevant to reconnection theories/models as they provide novel information on a process which is widespread in the universe.

CME-related CSs have been identified in SOHO UVCS spectra and in White Light data (see, e.g., Schwenn et al., 2006). SOHO UVCS revealed a bright, narrow feature in the [Fe XVIII] line at $974 \AA$ in the spectra of the 23 March 1998 CME (Ciaravella et al. 2002). The bright [Fe XVIII] emission was located between the loop arcade and the CME core and appeared in the aftermath of the CME ejection. Because the temperature of formation of Fe XVIII is on the order of $T=10^{6.7} \mathrm{~K}$, emission from this ion rarely appears even above active regions (ARs) and its detection was interpreted as indicative of a hot region, identified with the CS. Successive studies confirmed this interpretation (see e.g. Ko et al., 2003; Bemporad et al., 2006). CSs in LASCO WL data correspond to bright rays seen above cusp-like features associated with an arcade of loops that are reforming. Analogous ray-like features have been identified also in Solar Maximum Mission data in association with disconnection events related with CMEs and have been thoroughly discussed by Webb et al. (2003).

These recent works led Lin et al. (2007) to infer properties of CSs that had been observed in CMEs. To this end, Lin et al. selected three events that had been observed by UVCS and

Published by Copernicus Publications on behalf of the European Geosciences Union. 

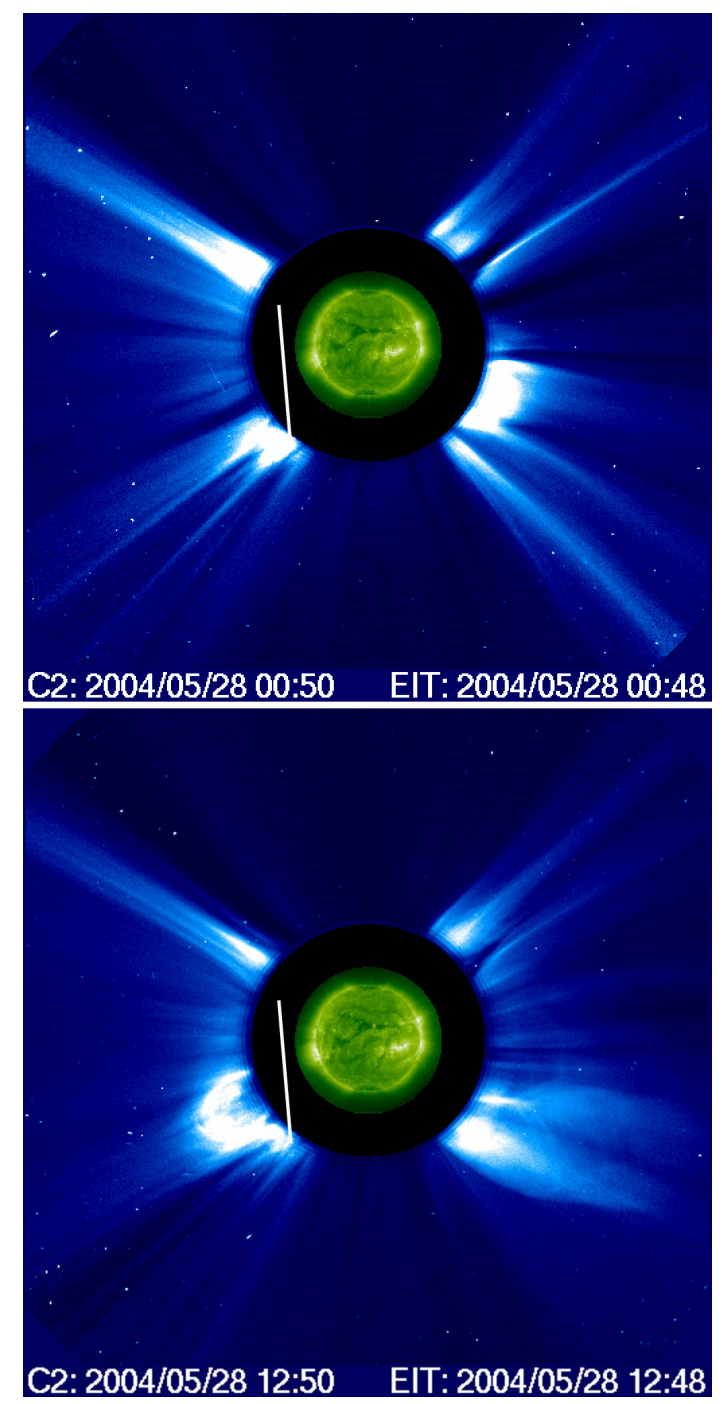

Fig. 1. Composite C2-EIT images of the corona: top, LASCO C2 image taken on 28 May 2004, at 00:50 UT, prior to the CME analysed in this paper; bottom, C2 image taken at 12:50 UT, about two hours after the CME initiation. EIT $195 \AA$ maps are shown at approximately the same times. The position of the UVCS slit is given in the images as a white bar, set normal to the radius of the Sun through a southern latitude of $4.8^{\circ}$, at an altitude of $1.55 R_{\odot}$, in the eastern hemisphere.

derived the CS thickness and resistivity. The latter turned out to be order of magnitudes higher than predicted for classical or anomalous resistivity. Presently, properties of reconnection in eruptive events have been inferred from flare observations, allowing one to derive the reconnection rate (see, e.g. Qiu et al., 2002) and the "bursty" character of reconnection (see, e.g. Fletcher et al., 2004). The new data provide further means to analyze the "observed" properties of reconnection and shed light on open issues.
Our work aims at adding to the information derived from CME CS studies by analyzing the slow event that occurred on 28 May 2004, at 11:06 UT, in the south eastern quadrant, for which we have LASCO and UVCS data. According to the LASCO CME catalog (Yashiro et al., 2004), this event occurred after an earlier CME, originating at 03:50 UT from a source slightly equatorward of the later CME source. In the following we identify CMEs by the time given in the LASCO catalog, i.e. by the time the CME front is first detected in LASCO images.

The paper is organized as follows: in Sect. 2 we describe UVCS and LASCO data and illustrate the evidence for reconnection in the two data sets. In Sect. 3 we derive the reconnection rate and give estimates of the CS resistivity, in the last section we briefly discuss our results and emphasize open issues.

\section{The observations}

\subsection{UVCS data}

Figure 1 shows the configuration of the corona on 28 May 2004, as it appears in LASCO C2 images prior to the event we analyzed (top panel) and about $1.5 \mathrm{~h}$ after the 11:06 CME that appears at a southern latitude of $\approx 16^{\circ}$, in the eastern hemisphere (bottom panel). The CME, reaching up to the $\mathrm{C} 2$ field of view, has a morphology consistent with traditional CME models (Hundhausen, 1987; Forbes, 2000). The line, normal to the solar radius, through a southern latitude of $4.8^{\circ}$, gives the position of the UVCS slit. The arch-like configuration of the CME is apparent also in Fig. 2, which shows an EIT (Extreme-Ultraviolet Imaging Telescope) difference image, built from data taken at 10:00 and 10:24 UT, where the event that initiated the CME is clearly identifiable. UVCS spectra, acquired at an heliocentric distance between those sampled by the EIT and LASCO experiment, should bear evidence of the CME as it passes through the UVCS slit.

Different UVCS grating positions were used between 00:49 UT and 11:34 UT and between 11:34 and 18:13 UT, both covering the $\mathrm{O}$ VI doublet lines at 1031.9 and $1037.6 \AA$ and the Si XII line at $520.7 \AA$ (observed in the second order). However, the slight different range of wavelengths covered by the two grating positions, enabled, from 00:49 UT to 11:34 UT, the observation of spectral lines originating from very hot plasmas, like the [Fe XVIII] $974.8 \AA$ line. Later on the lines with the highest formation temperature were those from $\mathrm{Ca} \mathrm{X}$ and $\mathrm{Al} \mathrm{XI}$ ions (at 557.8 and $550.0 \AA$, respectively, both observed in the second order), with maximum formation temperature of $10^{5.9}$ and $10^{6.2} \mathrm{~K}$, respectively. Data have been corrected for flat-fields effect and wavelength and radiometrically calibrated: uncertainties in radiometric calibration are within $20 \%$ for first order lines, but rise up to $50 \%$ for second order lines (Gardner et al., 2000). We also checked the 


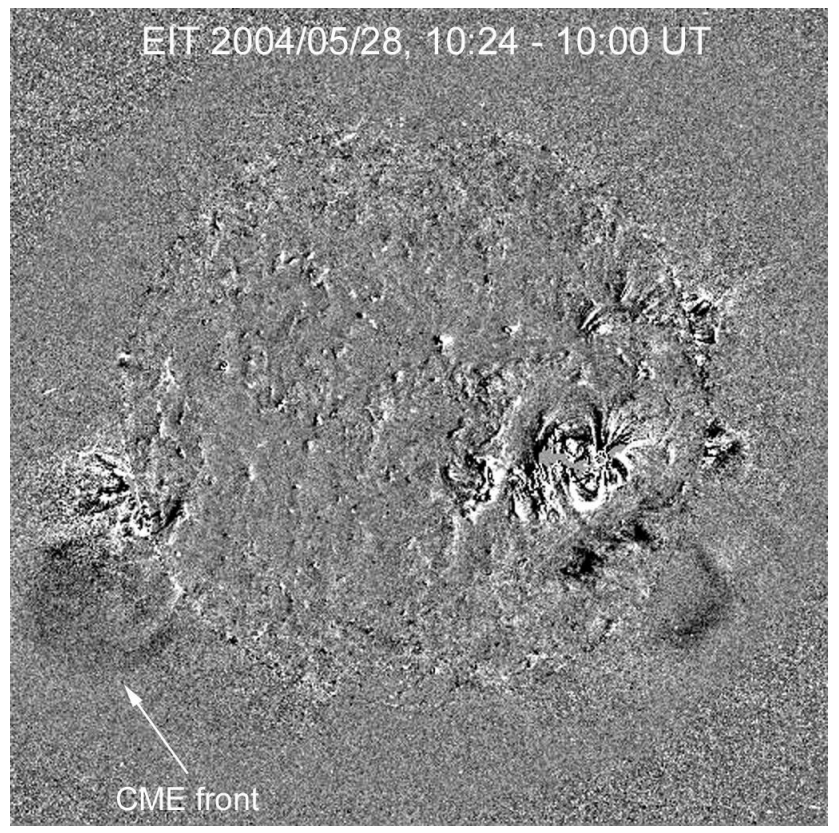

Fig. 2. EIT $195 \AA$ difference image on 28 May 2004, showing, in the south-eastern hemisphere, the initiation of the 11:06 UT CME at lower coronal levels than shown in LASCO images.

effect of stray light on the radiances of the $\mathrm{O}$ VI lines, that turned out to be irrelevant.

As mentioned earlier, CSs have been identified in UVCS data mainly from the presence of high emission from the Fe XVIII ion. This, however, is not the only means by which CSs have been identified, either because not all CSs show an enhanced [Fe XVIII] line emission, or because Fe XVIII lines were not within the wavelength range of spectra taken at the CME time. Alternative techniques include the observation of the Ly- $\alpha$ emission along the UVCS slit and its temporal evolution or the identification of the CME structure in difference maps in the $\mathrm{O}$ VI doublet lines or $\mathrm{H} \mathrm{Ly}-\alpha$ line. Lin et al. (2005) found a dark gap, whose width decreased in time, in the distribution of the Ly- $\alpha$ emission along the slit and ascribed it to the thinning of the hot CS in time. In O VI or Ly- $\alpha$ difference maps the successive transit through the slit of the CME front, bubble and core can be identified via an increased/decreased line emission that outlines the shape of the CME structures with a temperature and/or density slightly different than those of the ambient corona (see, e.g., Bemporad et al., 2007).

In this work we first looked for the [Fe XVIII] emission in the data taken with the grating position that included the $974 \AA$ line, but found no emission at that wavelength. Then we analyzed the behavior of the O VI $1032 \AA$ line radiance along the UVCS slit, at different times. In order to enhance the visibility of changes as a function of position and time, we subtracted the radiance of the line averaged over the

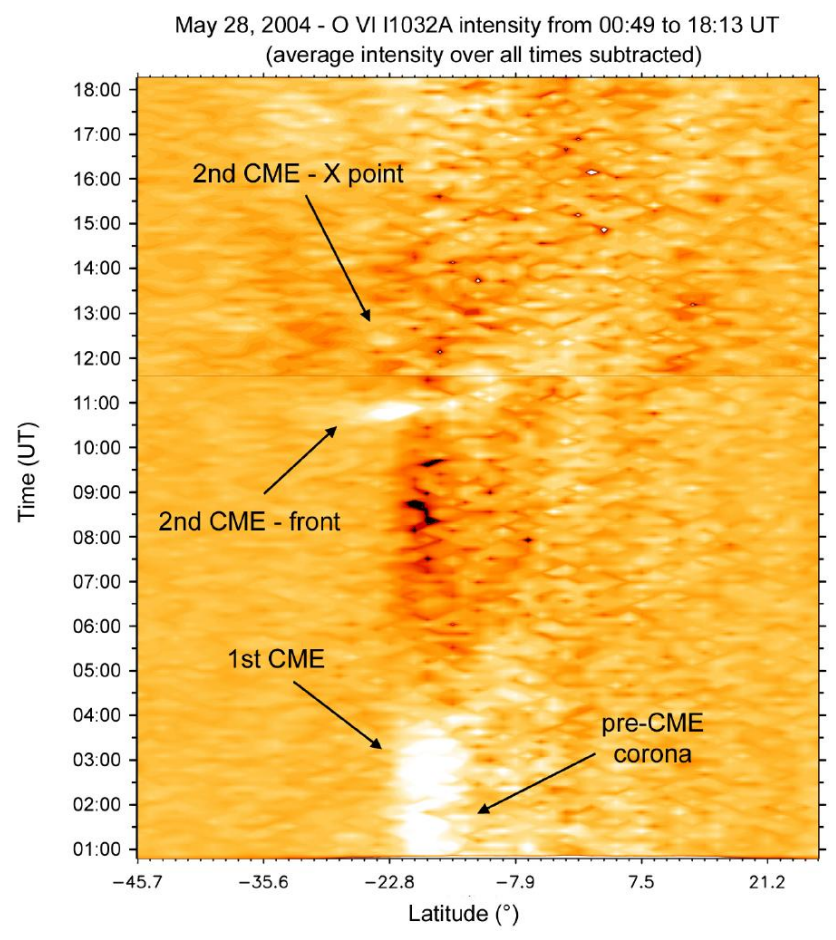

Fig. 3. Distribution of the intensity of the O VI $1032 \AA$ line as a function of the heliographic latitude (x-axis) and time (y-axis), derived from UVCS data. At each position along the slit, the intensity of the line averaged over the whole observing time, has been subtracted from individual intensities. The CME front reaching the UVCS slit at $\approx 10: 40$ UT and the reconnection point reaching the UVCS slit at $\approx 12: 20$ UT show up in the enhanced OVI 1032 brightness and are indicated by arrows.

whole time interval from individual radiances along the slit. Results from this procedure are given in Fig. 3.

The figure shows the radiance excess/deficit (bright/dark areas) of the O VI $1032 \AA$ line over its average value, as a function of position along the slit (x-axis, expressed in heliographic latitudes) and time (y-axis) over the time interval covered by UVCS observations. The bright area at the bottom of the figure (from $\approx 01: 00$ to $\approx 03: 30 \mathrm{UT}$ ) between southern latitudes $\approx 16-23$ degrees represents the enhanced emission from the active region at the East limb seen in Figs. 1, 2. Shortly after 03:00 UT, the front of the first CME (see Sect. 1) reaches the UVCS slit and crosses the slit at the positions where the AR emission had been detected. At later times, the dark area at approximately the same latitude, is a consequence of the CME transit. As the CME sweeps throught the slit, the coronal density is depleted and, as a consequence, the O VI $1032 \AA$ line radiance decreases. At about 10:40 UT a bright elongated patch, extending to more southern latitudes than the first CME, signals the transit of the front of the CME analyzed in this paper through the spectrograph slit. The O VI $1032 \AA$ line radiance increases by $\approx 30 \%$ over 


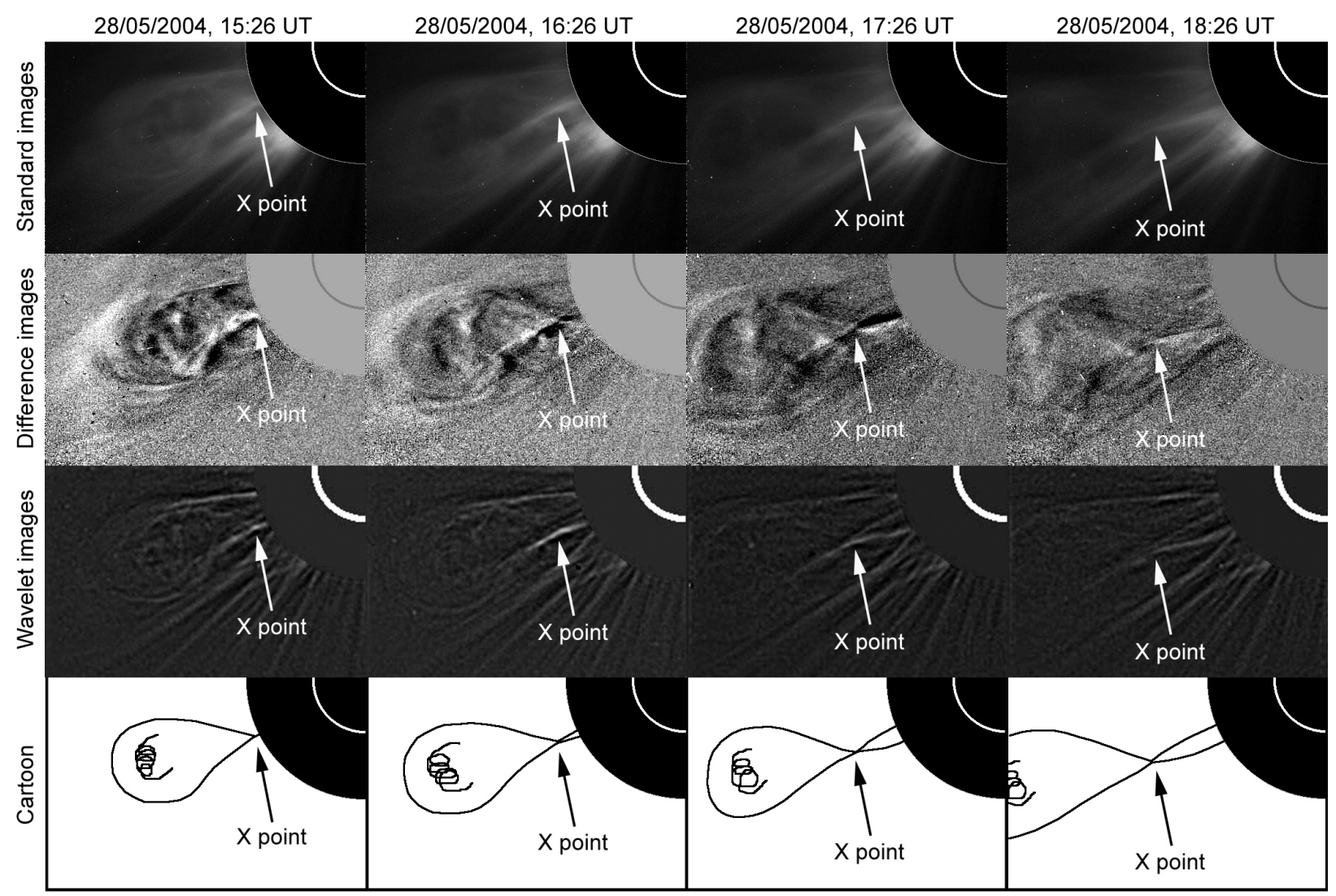

Fig. 4. Top row: LASCO C2 images at four times during the evolution of the CME which occurred on 28 May 2004, at 11:03 UT. Middle row, same as top row, for LASCO C2 difference images and, third row, same as above, for LASCO C2 wavelet enhanced images. White arrows, superposed onto the images, point to the position where two approaching features appear to merge. Merging occurs at increasing heliocentric distances with time. The cartoons in the bottom row help visualize the CME scenario identified in LASCO images.

the dark background and is only $\approx 15 \%$ lower than the active region emission. Roughly, assuming the $\mathrm{O}$ VI line to be collisionally excited, the increased radiance of the CME front corresponds to a density increase, over the region depleted by the earlier CME, by a factor 1.5-1.7 (assuming temperature and the extension along the line of sight occupied by the two events keep constant).

At later times, there is evidence of the transit through the slit, behind the front, at a latitude of $\approx 23^{\circ}$, of a small roundish bright feature, from which depart two bright branches. Its radiance is about $22 \%$ higher than the average brightness. Recalling the catastrophe model scenario (Lin and Forbes, 2000), we surmise that this bright feature corresponds to the site where reconnection occurs, below the CME bubble. Its position, and time of appearance, support our hypothesis: in this case, UVCS data reveal that reconnection reaches the $1.55 R_{\odot}$ level at $\approx 12: 20$ UT and we expect it progresses to increasingly higher altitudes as the CME propagates outwards. Admittedly, the UVCS evidence is not conclusive and we need further data to substantiate our claim. If our interpretation is correct we should find signatures of reconnection in LASCO C2 and $\mathrm{C} 3$ images.

\subsection{LASCO data}

The morphology of two-ribbon flares, where separating chromospheric ribbons trace the footpoints of a growing bright loop arcade, provides clues to the position where reconnection occurs. Other phenomena, like the loop shrinkage (see, e.g., Forbes and Acton, 1996), or magnetic field extrapolations that reproduce the configuration of the growing arcade, add further information that help us infer, at any time during the flare event, the level where reconnection occurs. Obviously, the situation is more difficult at the coronal levels imaged by the LASCO C 2 and $\mathrm{C} 3$ coronagraphs, where reconnection of weak fields is unlikely to result in features as easily observable as those in flares.

Some constraint may be provided by the detection of the diffusion region, which is supposedly located above the reconnecting arcade and below the CME. Figure 4 gives LASCO C2 images of the corona over a time interval of three hours, on 28 May 2004. In the top row, we give standard contrast enhanced images; difference images, at times corresponding to those in the upper row, are given in the middle row and wavelet enhanced images appear in the third row. 
In the bottom row a sequence of cartoons help visualize the scenario we inferred from the images.

The images in the top row, although rather fuzzy, show, below the expanding CME bubble, two approaching features that emerge from the dark occulter disk and converge at a level progressively higher in time: this shows up more clearly in the wavelet enhanced images (third row). Difference images - second row of Fig. 4 - as well as wavelet enhanced images, reveal the CME bubble overlying the level where the approaching features merged (well defined here as well) and, within the bubble, not far from the CME front, a dark area identifiable with the CME core. The sequence of Fig. 4 yields a scenario where merging features reconnect, forming an Xtype point, at increasingly higher levels below the evolving CME bubble. Further support to this interpretation comes from LASCO C 3 images. Figure 5 gives the CME configuration seen in the LASCO C 3 images acquired at 16:18 and 23:18 UT, on 28 May 2004. Below the CME bubble appears a "stem", possibly to be identified with the CS connecting the bubble to lower reconnecting features. Just above the occulter disk there is an admittedly non conclusive indication for the presence of a merging feature.

Summarizing the information we derived from LASCO observations, we conclude, from the analysis of standard or wavelet enhanced images, that this unique data set allowed us to trace the rise in time of an X-type reconnection point, assumed to be located at the level where two approaching features merge, throughout the $\mathrm{C} 2$ field of view. Difference images give results consistent with those derived from the original data. With increasing uncertainties, we also tried to identify the reconnecting point out into the $\mathrm{C} 3$ field of view: these latter estimates are only indicative and have an uncertainty which may well reach $\pm 1 r_{\odot}$. Above $\approx 4.5 R_{\odot}, \mathrm{C} 3$ images provide evidence of the formation of a CS reaching to the bottom of the CME bubble.

\section{Reconnection properties}

The identification in $\mathrm{C} 2$ and $\mathrm{C} 3$ images of the reconnection point, hereafter referred to as the X-point, over a long time interval, allows us to plot its rise with time through the LASCO field of view and to complement these data with the UVCS data point at a lower level. Figure 6 gives the temporal profiles of the CME front, core, and X-point, in the range between 1.55 and $12-14 R_{\odot}$. The CME front and core rise with a speed of $130 \mathrm{~km} / \mathrm{s}( \pm 20 \mathrm{~km} / \mathrm{s})$ up to $5-6 R_{\odot}$ and accelerate to about $200 \mathrm{~km} / \mathrm{s}( \pm 20 \mathrm{~km} / \mathrm{s})$ at larger altitudes. The latter value agrees with the speed $(205 \mathrm{~km} / \mathrm{s})$ given by the LASCO catalog on the basis of a data set which gets up to $23 R_{\odot}$. The $\mathrm{X}$-point rises more slowly at an average speed of $65 \mathrm{~km} / \mathrm{s}$ over the height interval between 1.5 and $5 R_{\odot}$. The points at $1.55 R_{\odot}$ in the height vs. time plots of the CME front and X-point have been derived from the UVCS data and lie on the linear extrapolation to earlier times of

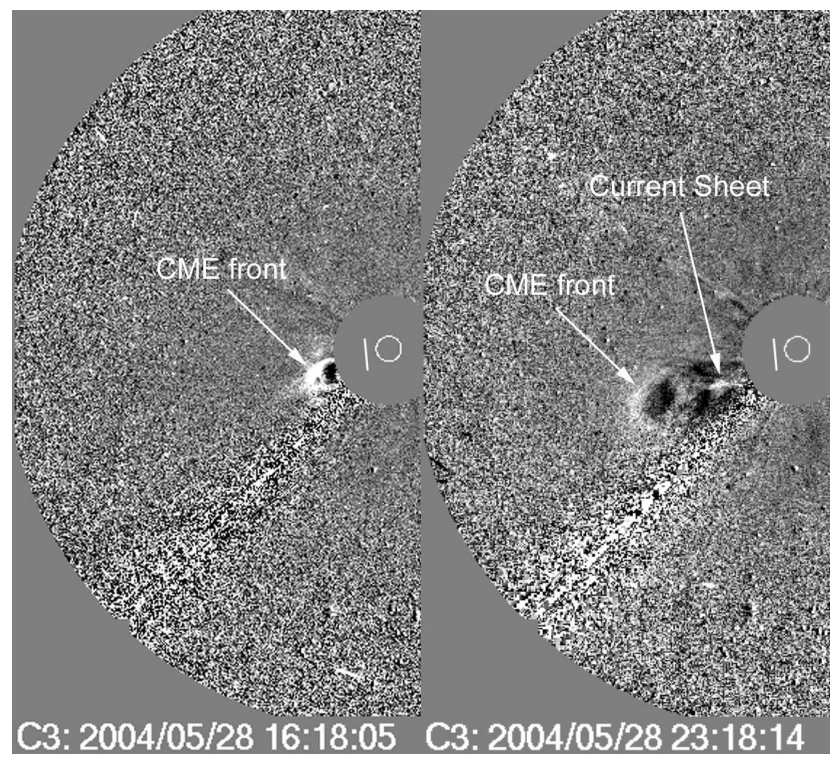

Fig. 5. LASCO C3 running difference images at 16:18 and 23:18 UT, on 28 May 2004, showing the morphology of the CME at a time when the CME front and bubble are seen in the $\mathrm{C} 3$ field of view.

the LASCO profiles (to this end we obviously consider only the profile of the CME front at times earlier than 16:00 UT when the slope of the curve changes). A linear backward extrapolation should be accurate enough, because the speed of the front between the levels sampled by EIT and by UVCS turns out to be $\approx 120 \mathrm{~km} / \mathrm{s}$, in good agreement with the speed evaluated from the LASCO data. This supports the linear backward extrapolation of the X-point. The difference in the rise speed of the front, with respect to the $\mathrm{X}$-point, originates from the elongation of the CME bubble in time, as usually observed. We conclude that the UVCS points nicely fit the LASCO data and put more weight on the interpretation we suggested in Sect. 2. To our knowledge this is the first time that the rise of the X-point is followed up to these large heliocentric altitudes.

The rise of the $\mathrm{X}$-point is crudely indicative of the rate at which open magnetic field lines are swept into the diffusion region, reconnect and close down. The reconnection rate $M_{0}$ is defined as the rate at which open magnetic flux is convected into the diffusion region where the merging takes place, (or, equivalently, the rate at which closed magnetic flux appears as the neutral line rises). $M_{0}$, however, is not usually given in Maxwell/s but as an adimensional number that gives the ratio of the plasma inflow speed $v_{i}$ (inflow towards the diffusion region) to the Alfvén speed $V_{A}$ outside the diffusion region $M_{0}=v_{i} / V_{A}$. The reconnection rate has first been evaluated about 20 years ago by Poletto and Kopp (1986), in two-ribbon flares. Over the past few years, the reconnection rate in flares has been derived by many authors, 

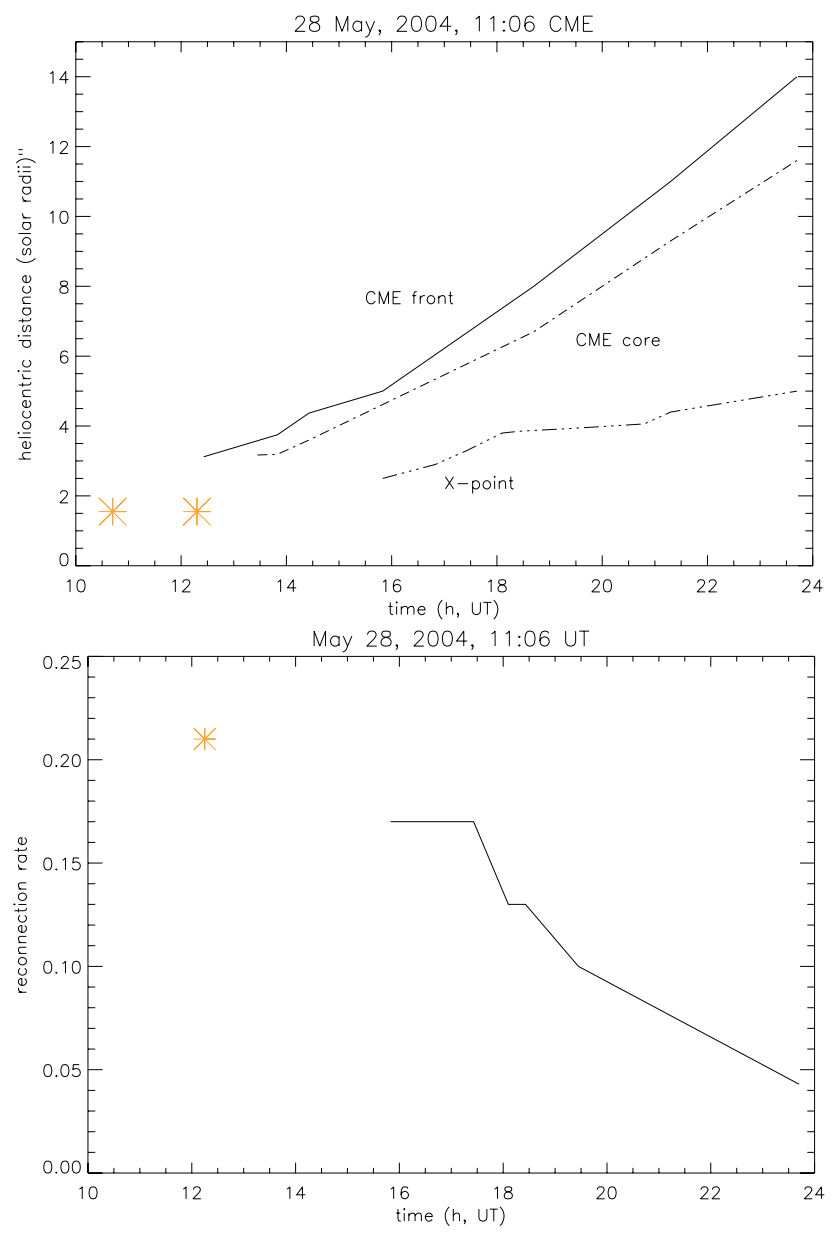

Fig. 6. Top panel: rise with time of the CME front and core (solid and dot-dashed line, respectively) and of the X-point; bottom panel: temporal profile of the reconnection rate from 1.55 to $5 R \odot$. The star symbols refer to data points derived from UVCS observations and show the times at which the CME front $(\approx 10: 40 \mathrm{UT})$ and the X-point $(\approx 12: 20 \mathrm{UT})$ reach the $1.55 R_{\odot}$ level where the UVCS slit was set. The data point have an uncertainty lower than a factor 2 .

(see, e.g., Fletcher and Hudson, 2002; Qiu et al., 2002; Isobe et al., 2005), but there are very few measures of $M_{0}$ in the diffusion region below CMEs. At the high heliocentric distances where typically CMEs are observed, it is quite difficult to give plausible estimates of the magnetic field that enters the expression of $V_{A}$ as well as to measure plasma inflows.

Ko et al. (2003) and Lin et al. (2005) solved these problems by measuring outflows from the CME associated diffusion region and, in agreement with the standard theory of magnetic reconnection, assumed $V_{A}$ to be equal to the outflow speed (Priest and Forbes, 2000). Lin et al. (2007) inferred an inflow speed $\leq 10 \mathrm{~km} / \mathrm{s}$, at $4-5 R_{\odot}$ from the changes in the width of the $\mathrm{Ly}_{\alpha}$ gap along the UVCS slit (see Sect. 2.1). Previous evidence of inflows was presented by Yokoyama et al. (2001) and Narukage and Shibata (2006): these estimates range between 2 and $15 \mathrm{~km} / \mathrm{s}$, but refer to inflows in flare events. Typical values of $M_{0}$ in flares range between 0.001 and 0.1 and between 0.01 and 0.23 in CMEs. Here we aim at inferring the reconnection rate in the CME of 28 May 2004, thus adding to the few CME related $M_{0}$ values.

It is well known that in the Sweet-Parker reconnection regime (Sweet, 1958; Parker, 1957, 1963) the reconnection rate $M_{0}=R_{m}^{-1 / 2}$ (where $R_{m}=\mu V_{A} L / \eta$ is the Lundquist number, $\eta$ is the magnetic diffusivity, $L$ the length of the CS, $\mu$ the plasma permeability) turns out to be too slow to account for the fast energy release in flares. Petschek (1964), invoking a much more localized diffusion region and two pairs of slow mode standing shocks (SMSS) that accelerate the plasma not passing through the diffusion region, derived a reconnection rate $\left(M_{0} \propto\left(\ln R_{m}\right)^{-1}\right)$ appropriate to the observed timescale of the flare energy release. In the steady-state 2-D Petschek reconnection, a pair of standing slow mode shocks bound the jets outflowing from the diffusion region along the $\mathrm{CS}$. The included angle in between the slow mode shocks extending from the diffusion regions is $2 \theta$, where $\theta$ is the angle with respect to the axis of symmetry (i.e. with respect to the outflow direction). The angle $\delta$ in between the SMSS and the inflowing field line is small (Aurass et al., 2002), hence $\theta$ is a good approximation of the angle between the inflowing field line and the axis of symmetry of the system. The continuity equation across the SMSS yields, for a solar wind speed $v_{\text {out }} \ll V_{A}$ and for an incompressible plasma a reconnection rate $\tan \theta \approx M_{0}$ (see, e.g., Vršnak and Skender, 2005). Here we identify the bright UVCS and LASCO features converging into the X-point as the SMSS waves and infer the reconnection rate by measuring at different times the angle $2 \theta$ they form.

The results from this procedure are shown in Fig. 6, bottom panel. Although values derived on the basis of these simplistic assumptions are expected to provide only a crude indication of true reconnection rates, the figures we obtained are in good agreement with the few values given in the literature (see the Lin et al., 2007, paper mentioned above). We may give an upper limit of a factor 2 to the uncertainties in the measurements of the angle in between the bright features seen in LASCO images, hence this is also an upper limit to the values of the rate we inferred.

The rate decreases by a factor $\approx 4$ in between 1.55 and $7 R_{\odot}$, i.e., it decreases linearly with increasing altitudes. Uncertainties in the angle measurement do not have a major impact on the reconnection rates, which still may be considered typical of the Petschek regime. If we compare Fig. 4, where there is no indication of the formation of a CS, with Fig. 5, where a CS appears, we may tentatively propose that the decrease in the reconnection rate hints to an evolution of the system from a fast Petschek-like to a slower Sweet-Parker reconnection.

We now turn to the CS properties and give an estimate of its resistivity. To this end we need to know densities in the 
CS and inflows towards the CS. First we evaluate the density in the CS and in nearby regions, under the assumption of spherical symmetry, from the LASCO C $3 \mathrm{pB}$ map, acquired at 22:55 UT. At that time we know, from Fig. 5, that the CS extends from above $\approx 4.5$ out to $\approx 6-7 R_{\odot}$. Because the assumption of spherical symmetry is likely inadequate for a CS, we also derived densities assuming a CS whose length along the line of sight (LOS) equals its width on the plane of the sky. The inferred densities represent the lower and upper limits to the CS densities. Figure 7 shows that densities in the CS above $4.5 R_{\odot}$ are at least $10-30 \%$ higher than the undisturbed corona.

As we said, at large heliocentric distances the detection and measurements of inflows towards the CS are extremely difficult. Values of inflows, coupled with measurements of the CS thickness, allow one to compute the "observed" values of resistivity, which is given, in the hypotheses listed above, by $\eta_{e}=\eta \mu=v_{i} \delta \mu$ (where $\delta$ is the CS half thickness and $\eta_{e}$ is in ohm m). With $v_{i}$ on the order of $\approx 1-10 \mathrm{~km} / \mathrm{s}$ (in agreement with available measurements) and the thickness of the diffusion region measured on LASCO images $(\delta$ $\approx 5 \times 10^{4}-5 \times 10^{5} \mathrm{~km}$, in agreement with measurements by Ko et al., 2003, and Lin et al., 2007), the "observed" resistivity $\eta_{e}$ ranges between $6 \times 10^{4}$ and $6 \times 10^{6} \mathrm{ohm} \mathrm{m}$. Lin et al. (2007) inferred an observed resistivity for the three events they analyzed on the order of $5 \times 10^{5} \mathrm{ohm} \mathrm{m}$, midway between our lower and upper limits.

The classical resistivity depends weakly on temperature (Priest, 1982): $\eta_{c}=4 \pi \times 10^{2} T^{-3 / 2} \mathrm{ohm} \mathrm{m}$. Assuming a temperature $T$ on the order of $T=1 .-5 . \times 10^{6} \mathrm{~K}$ we get a classical resistivity $\eta_{c}$ on the order of $10^{-5}-10^{-6} \mathrm{ohm} \mathrm{m}$, about ten orders of magnitude lower than the "observed" value. We next check whether anomalous resistivity, arising from the particle wave interaction, is high enough to reproduce the observed resistivity. The anomalous resistivity $\eta_{a}$ is inversely proportional to the square root of density (Priest, 1982, $\eta_{a}=6.4 \pi 10^{6} N_{e}^{-1 / 2} \mathrm{ohm} \mathrm{m}$ ): taking the lower and upper limits to the densities inferred at $5 R_{\odot}$ we derive $10 \leq \eta_{a} \leq 60 \mathrm{ohmm}$. Even if quite higher than the classical resistivity, the $\eta_{a}$ values are far below the "observed" resistivity.

\section{Discussion and open issues}

LASCO coronagraph images of the slow CME that occurred on 28 May 2004 at 11:06 UT constitute quite an unusual data set, as they offer the opportunity of following the rise of the reconnection point over many hours, hence as it rises to large heliocentric distances. This allowed us to derive the profile of the rate of reconnection $M_{0}$ with heliocentric distance: to our knowledge this is the first time that such a profile is shown. Because the assumption we made to infer $M_{0}$ are rather crude, we place more weight on the trend shown by the reconnection rate than on the absolute values we inferred.

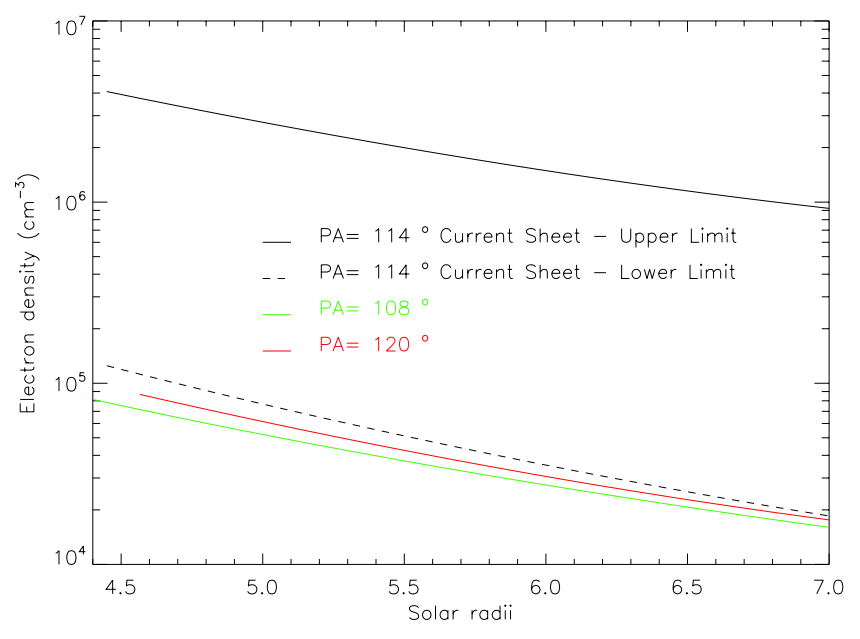

Fig. 7. Profile of the CS density vs. heliocentric distance, derived from the LASCO $\mathrm{pB}$ map at 22:55 UT, under the assumption of spherical symmetry (dotted line) and under the assumption of a CS length (along the LOS) equal to the CS width on the plane of the sky (solid line). Green and red lines give coronal densities in regions adjacent to the CS.

However, we surmise that the decrease of $M_{0}$ possibly hints to a transition from the Petschek to the Sweet-Parker regime, for which we have scarce observational evidence, so far.

The issue of the transition from fast to slow reconnection has been discussed in several theoretically oriented papers (see, e.g., Oreshina and Somov, 1998; Somov and Oreshina, 2000). Recently Nitta (2007), in a paper where he investigates 2-D MHD reconnection solutions over a large range of magnetic Reynolds numbers, also questioned the observational estimates of the reconnection rate from the inflow speed, on the ground that this may be appropriate only to the case when the reconnection point is at rest in the initial plasma rest frame. Clearly, this issue and the search for any observational evidence of the transition between diverse reconnection regime need to be thoroughly pursued.

We have also shown that we need to understand the nature of the resistivity in CSs. The high 'observed' values call for a highly turbulent CS. Possibly enhanced resistivity may be provided by the tearing instability that most likely develops in CSs and may be responsible for an hyperresistivity (see Lin, 2007, and references therein). The identification of the processes responsible for the CS resistivity is, as of today, a further open topic

A more general question we may pose is about the meaning of the parameters we inferred. Are these averages over an ensemble of mini CSs that constitute the macroscopic CS we observe? Karlický et al. (2005) examined power spectra of structures observed during flares in the 500 to $1500 \mathrm{MHz}$ range and found that in the 0.06 to $0.2 \mathrm{~s}$ range the spectra followed a power law. Is this pointing to fractal structure of 
CSs? This possibility may lead to a behavior of individual unobservable CSs that should be compatible with the overall values observationally derived. We expect this as well to be an active field of research in the near future.

Finally, we like to mention briefly a couple of alternative interpretations to the data set we analyzed. First, we consider whether the bright point we identified with the X-point, might be ascribed to a rising twisted loop system, the brightest area originating as a consequence of the enhanced brightness where the twist intercepts the observer LOS. This interpretation is a priori plausible.

However, Fig. 4 shows clearly the CME bubble and we expect reconnection to occur below it. It seems to us highly coincidental that a system of loops rises, keeping its brightest region under the bubble, from the $1.55 R_{\odot}$ level out to distances of several solar radii: loop systems do not usually reach such high heliocentric distances. For instance, the so-called giant arches that form after flares/CMEs (see, e.g., Švestka et al., 1995; Tsuneta, 1996) reach altitudes of 0.2$0.3 R_{\odot}$ rising with a speed on the order of $10 \mathrm{~km} / \mathrm{s}$. Here we should invoke larger speeds and a rise to much larger altitudes. It looks like this might be a possibility to be examined only when lacking more straightforward interpretations.

Another possibility that we can't dismiss, questions the identification of a CS at the distances probed by the LASCO C3 coronagraph. We might as well hypothesize that we are observing the region compressed in between the SMSS. In a scenario where the reconnection rate is decreasing in time, we expect the angle in between the SMSS to decrease and eventually become too small to be identified. If this is the case, the reconnection rate will decrease out to larger distances than given in Fig. 6. This is a further open issue that needs to be addressed in future.

Acknowledgements. A. B. and G. P. thank ISSI (International Space Science Institute, Bern) for the hospitality provided to the members of the team on the Role of Current Sheets in Solar Eruptive Events, where many of the ideas presented in this work have been discussed. A. B. and G. P. acknowledge support from ASI/INAF I/035/05/0 and ASI/INAF I/05/07/0. SOHO is a project of international collaboration between ESA and NASA. The LASCO images have been enhanced using a technique developed by Guillermo Stenborg.

Topical Editor R. Forsyth thanks two anonymous referees for their help in evaluating this paper.

\section{References}

Aurass, H., Vršnak, B., and Mann, G.: Shock-excited radio burst from reconnection outflow jet?, Astron. Astrophys., 384, 273$281,2002$.

Antiochos, S. K., DeVore, C. R., and Klimchuck, J. A.: A model for Solar Coronal Mass Ejections, Astrophys. J., 510, 485-493, 1999.

Bárta, M., Vršnak, B., and Karlický, M.: Dynamics of plasmoids formed by the current sheet tearing, Astron. Astrophys., 477, 649-655, 2008.
Bemporad, A., Poletto, G., Suess, S. T., et al.: Current Sheet evolution in the aftermath of a CME event, Astrophys. J., 638, 11101128, 2006.

Bemporad, A., Raymond, J. C., Poletto, G., et al.: A comprehensive study of the initiation and early evolution of a Coronal Mass Ejection from Ultraviolet and White-Light data, Astrophys. J., 655, 576-590, 2007.

Ciaravella, A., Raymond, J. C., Li, J., et al.: Elemental abundances and Post-Coronal Mass Ejection Current Sheet in a very hot Active Region, Astrophys. J., 575, 1116-1130, 2002.

Fletcher, L. and Hudson, H.: Spectral and spatial variations of flare Hard X-ray footpoints, Solar Phys., 210, 307-321, 2002.

Fletcher, L., Pollock, J. A., and Potts, H. E.: Tracking of TRACE ultraviolet flare footpoints, Solar Phys., 222, 279-298, 2004.

Forbes, T. G.: A review on the genesis of coronal mass ejections, J. Geophys. Res., 105, 23 153-23 166, 2000.

Forbes, T. G. and Acton, L. W.: Reconnection and field line shrinkage in solar flares, Astrophys. J., 459, 330-341, 1996.

Gardner, L. D., Atkins, N., Fineschi, S., et al.: Efficiency variations of UVCS/SOHO based on laboratory measurements of replica gratingsi, Proc. SPIE, 4139, 362-369, 2000.

Hundhausen, A. J.: The origin and propagation of Coronal Mass Ejections, in: Sixth International Solar Wind Conference, edited by: Pizzo, V. J., Holzer, T., Sime, D. G., 181 p., 1987.

Isobe, H., Takasaki, H., and Shibata, K.: Measurements of the energy release rate and reconnection rate in solar flares, Astrophys. J., 632, 1184-1195, 2005.

Karlický, M., Bárta, M., Mészárosová, H., et al.: Time scales of the slowly drifting pulsating structure observed during the April 12, 2001 flare, Astron. Astrophys., 432, 705-712, 2005.

Ko, Y.-K., Raymond, J. C., Lin, J., et al.: Dynamical and physical properties of a post-Coronal Mass Ejection Current Sheet, Astrophys. J., 594, 1068-1084, 2003.

Lin, J. and Forbes, T. G.: Effects of reconnection on the coronal mass ejection process, J. Geophys. Res., 105, 2375-2392, 2000.

Lin, J., Ko, Y.-K., Sui, L., et al.: Direct observations of the magnetic reconnection site of an eruption on 2003 November 18, Astrophys. J., 622, 1251-1264, 2005.

Lin, J., Li, J., Forbes, T. G., et al.: Features and properties of Coronal Mass Ejection/Flare current sheets, Astrophys. J., 658, L123126, 2007.

Narukage, N. and Shibata, K.: Statistical analysis of reconnection inflows in solar flares observed with SOHO EIT, Astrophys. J., 637, 1122-1134, 2006.

Nitta, S.-Y.: Continuous transition from fast magnetic reconnection to slow reconnection and change of the reconnection system structure, Astrophys. J., 663, 610-624, 2007.

Oreshina, A. V. and Somov, B. V.: Slow and fast magnetic reconnection. I. Role of radiative cooling, Astron. Astrophys., 331, 1078-1086, 1998.

Parker, E. N.: Sweet's mechanism for merging magnetic fields in conducting fluids, J. Geophys. Res., 62, 509-520, 1957.

Parker, E. N.: The solar-flare phenomenon and the theory of reconnection and annihiliation of magnetic fields, Astrophys. J. Supplement, 8, 177-211, 1963.

Petschek, H. E.: Magnetic field annihilation, in: AAS-NASA Symposium on the Physics of solar flares, NASA-SP50, edited by: Hess, W. N., 425 p., 1964.

Poletto, G. and Kopp, R. A.: Large-scale electric fields resulting 
from magnetic reconnection in the corona, in: The lower Atmosphere of Solar Flares, edited by: Neidig, D. F., 453 p., 1986.

Priest, E. R.: Solar magneto-hydrodynamics, Dordrecht, Holland; Boston: D. Reidel Pub. Co., p. 79-80, 1982.

Priest, E. T. and Forbes, T. G.: Magnetic reconnection, Cambridge University Press, Cambridge, UK, p. 122, 2000.

Qiu, J., Lee, J., Gary, D. E., et al.: Motion of flare footpoint emission and inferred electric field in reconnecting current sheets, Astrophys. J., 565, 1335-1347, 2002.

Riley, P., Lionello, R., Mikić, Z., et al.: Bursty reconnection following solar eruptions: MHD simulations and comparison with observations, Astrophys. J., 655, 591-597, 2007.

Schwenn, R., Raymond, J. C., Alexander, D., et al.: Coronal Observations of CMEs, Space Sci. Rev., 123, 127-176, 2006.

Somov, B. V. and Oreshina, A. V.: Slow and fast magnetic reconnection. II. High-temperature turbulent-current sheet, Astron. Astrophys., 354, 703-713, 2000.

Švestka, Z., Fàrnik, F., Hick, P., et al.: Large-scale active coronal phenomena in YOHKOH SXT images, I, Solar Phys., 161, 331363, 1995.
Sweet, P. A.: The neutral point theory of solar flares, in: Electromagnetic phenomena in Cosmical Physics, IAU Symp. No 6, edited by: Lehnert, B., 123 p., 1958.

Tsuneta, S.: Structure and dynamics of magnetic reconnection in a solar flare, Astrophys. J., 456, 840-849, 1996.

Vršnak, B. and Skender, M.: 2 1/2-Dimensional reconnection model and energy release in solar flares, Solar Phys., 226, 97119, 2005.

Wagner, W. J.: Coronal Mass Ejections, Annual Review Astronomy and Astrophysics, 22, 267-289, 1984.

Webb, D. F., Burkepile, J., Forbes, T. G., et al.: Observational evidence of new current sheets trailing coronal mass ejections, J. Geophys. Res., 108(A12), 1440, doi:10.1029/2003JA009923, 2003.

Yashiro, S., Gopalswamy, N., Michalek, G., et al.: A catalog of white light coronal mass ejections observed by the SOHO spacecraft, J. Geophys. Res., 109(A7), A07105, doi:10.1029/2003JA010282, 2004.

Yokoyama, T., Akita, K., Morimoto, T., et al.: Clear evidence of reconnection inflow of a solar flare, Astrophys. J., 546, L69-72, 2001. 